\title{
Manuscritos y Humanismo en los libros de caballerías: la materialidad en la ficción
}

\author{
Daniel GutiÉrrez TrÁPAgA \\ Universidad Nacional Autónoma de México \\ dgtrapaga@hotmail.com
}

La presencia de los manuscritos en los múltiples universos de ficción en los libros de caballerías ha contribuido al estudio de diversos temas como la magia, los personajes, las estructuras narrativas, los tópicos y motivos del género, aspectos que aparecen empleados o parodiados inclusive en los Quijotes, tanto los de Cervantes como el de Alonso Fernández de Avellaneda. Dos tipos de libros manuscritos se destacan en los relatos de caballerías de los siglos XVI y XVII: los de magia o grimorios y los de crónicas o historias fingidas. Estos últimos, los que sustentan la pseudohistoricidad de los libros de caballerías a partir de los tópicos del manuscrito encontrado y la falsa traducción, son el objeto de estudio particular de este trabajo (Eisenberg 1974; Marín Pina 1994). Si bien estos tópicos no son novedosos en la historia de la literatura y se remontan a la Antigüedad y la Edad Media (García Gual 1996), son un rasgo de identidad del género que muestra características de la cultura textual de la época y la diversidad presente en los libros de caballerías.

El presente estudio no pretende hacer una revisión exhaustiva de los más de ochenta textos del género que han sobrevivido hasta nuestros días, sino centrarse en algunos ejemplos que describen con cierto detalle los libros manuscritos, en tanto que objetos, y su historia ficticia ${ }^{1}$. Para ello,

* Este trabajo se realizó en el marco y con financiamiento del Proyecto PAPIIT (núm. IN405919), «La construcción narrativa en los ciclos de caballerías hispánicos» de la Dirección General de Asuntos del Personal Académico y de la Facultad de Filosofía y Letras de la Universidad Nacional Autónoma de México. Asimismo, es parte de las actividades del Seminario de Estudios sobre Narrativa Caballeresca (SEM/01_011_2019) de la misma Facultad.

${ }^{1}$ En varios casos, hay descripciones muy parcas que casi no desarrollan ni los rasgos, ni la historia de los libros que se supone traducen. Algunos de ellos ni siquiera remiten a la materialidad de los libros, sino al puro texto y sus aspectos lingüísticos, como ocurre en el caso del Florambel de Lucea: «Este libro fue traduzido de la lengua inglesa en la nuestra castellana, y agora nuevamente fue trasladado, corregido y emendado por Enciso» (Enciso Zarate 2009: 3); en el Primaleón: «Fue trasladado este segundo libro de Palmerín, llamado Primaleón, y ansimesmo el primero, llamado Palmerín, de griego en nuestro lenguaje castellano y corregido y emendado» (Marín Pina 1998: 538). En este caso, se recurre al tópico en un lugar inusual, el colofón, cuando normalmente se plantea en alguna de las distintas instancias prefaciales. Este hecho lleva a pensar que en el caso del Primaleón, la presencia del tópico de la falsa traducción busca simplemente cumplir de manera mínima con el horizonte de expectativas del género. 
siguiendo los principios de la bibliografía material, el trabajo se enfoca en los rasgos materiales que caracterizan a los manuscritos, incluyendo formato y estado de conservación. De igual manera, se tomará en cuenta la historia de la producción del objeto y de la transmisión y recepción de estos manuscritos presentados en la ficción (McKenzie 2004: 12).

En particular, este trabajo busca mostrar cómo en los textos seleccionados estos factores aparecen presentados en el tópico del manuscrito encontrado y la falsa traducción en términos afines a los temas, preocupaciones y labores filológicas propias del Humanismo. Es decir, la representación material de los manuscritos de los libros de caballerías aquí estudiados, tanto en su caracterización física como en su historia, se da en términos explícitamente afines a los rasgos del Humanismo. Para ello, se compararán las características y procesos de los manuscritos de la ficción con los que definieron al Humanismo: «It involves above all the rediscovery and study of ancient Greek and Roman texts, the restoration and interpretation of them and the assimilation of the ideas and values they contain» (Mann 1996: 2). Así, se mostrará cómo los libros de caballerías elegidos plantean, siguiendo al Humanismo, el descubrimiento y rescate de antiguos manuscritos, casi siempre griegos y latinos, junto con la traducción y la edición de dichas obras. De igual manera, el género de ficción remite a una serie de autores grecolatinos y humanistas para explicar los supuestos manuscritos. Es decir, se propone, siguiendo a McKenzie, que la manera de presentar los libros en la ficción corresponde a los significados de una realidad social vinculada a las prácticas y a la realidad material del Humanismo (2004: 13-15).

Gran parte de los autores humanistas, desde Petrarca hasta Vives, por mencionar a los más obvios, expresaron diversas quejas en contra de la novela artúrica y los libros de caballerías (Sarmati 1996). A pesar de lo anterior, muchos autores de libros de caballerías conocían y compartían las ideas e intereses del Humanismo, inclusive al punto de escribir libros de caballerías atendiendo las objeciones de esta corriente de pensamiento, como en el caso del Florisando de Páez de Ribera (Gutiérrez Trápaga 2017), o responder explícitamente a dichas críticas, como el prólogo de Ortúñez de Calahorra a su Espejo de príncipes y caballeros. A pesar de las críticas al género, el pensamiento del Humanismo comparte con los tópicos de los libros de caballerías el interés por los viejos libros manuscritos:

There was no humanism without books. They were the prime material on which the movement was founded and the natural medium through which it was transmitted. All humanists were consumers, and usually also producers, of books in manuscript. Many humanists first gained a reputation by seeking out and accumulating books. [...] Throughout the

Además, se hace extensivo al Palmerín de Olivia, texto que carece de explicación sobre el origen de su historia. 
Renaissance, secular and ecclesiastical princes with cultural pretensions built themselves up with libraries as much as any other trappings of civilization. A book was often the vehicle of an alliance between culture and power, in the form of translations or dedications of original works, commissioned or unsolicited. (Davies 1996: 47)

Así, se mostrará cómo en una parte del corpus de los libros de caballerías castellanos la presencia del manuscrito encontrado y la falsa traducción se plantea en términos afines al trabajo filológico del Humanismo y su realidad social. Si bien gran parte de la circulación del género se dio gracias a la imprenta, su realidad diegética apunta al mundo manuscrito y humanista, ambos de gran presencia contemporánea.

\section{AMADÍS DE GAULA Y SU CICLO}

La obra fundacional y paradigmática del género recurre, como es sabido, al tópico del manuscrito encontrado y la falsa traducción desde su prólogo. Es en dicho pasaje donde se dan detalles sobre el libro que supuestamente sirvió de fuente a la obra de Rodríguez de Montalvo:

corrigiendo estos tres libros de Amadís, que por falta de los malos escriptores, o componedores, muy corruptos y viciosos se leían, y trasladando y enmendando el libro cuarto con las Sergas de Esplandián su hijo, que hasta aquí no es en memoria de ninguno ser visto, que por gran dicha paresció en una tumba de piedra, que debaxo de la tierra en una hermita, cerca de Constantinopla fue hallada, y traído por un úngaro mercadero a estas partes de España, en letra y pargamino tan antiguo, que con mucho trabajo se pudo leer por aquellos que la lengua sabían; en los cuales cinco libros como quiera [...] (Rodríguez de Montalvo 1987: 224-225)

El pasaje da pormenores del estado del texto, el objeto y su historia con una serie de elementos significativos para su contexto y para el programa ideológico de Montalvo. En primer lugar, se presenta un texto dañado a raíz de la intervención incompetente del texto, así como la novedad de una historia recién descubierta: la continuación de la obra, las Sergas. En segunda instancia, se atiende al lugar de descubrimiento del texto: un espacio santo y cercano al centro geográfico de la trama de las Sergas: Constantinopla, a cuyo trono asciende Esplandián ${ }^{2}$. En tercer lugar, se enfatiza la antigüedad del texto, su soporte y un estado de conservación pobre, que si bien no se detalla, sí queda claro que dificulta su lectura.

${ }^{2}$ Los referentes geográficos, al igual que la presencia de estas crónicas al interior de la ficción, que remiten a la realidad extratextual generan complejas estructuras ficcionales de mise en abyme que son características del género (Demattè 2005). 
El primer aspecto, la corrupción textual, permitió a Rodríguez de Montalvo justificar su reescritura de las versiones medievales del Amadis y ofrecer la propia. Dicha preocupación se vincula directamente con la clasificación genérica que el autor medinés hizo de su obra: la historia fingida (Fogelquist 1982: 9-43; Bognolo 1998). Esta categoría pertenece a una de tres que se presentan en el prólogo, junto con la historia verdadera y la amplificada. Más allá de las diferencias entre dichos géneros, en cualquier caso el valor recae en su capacidad didáctica en términos cristianos: «¿qué tomaremos de las unas y otras, que algún fruto provechoso nos acarreen? Por cierto, a mi ver, otra cosa no salvo los buenos enxemplos y doctrinas que más a la salvación nuestra se allegare [...]» (Rodríguez de Montalvo 1987: 223). Luego, la corrupción textual resulta en una versión que no está a la altura de su contenido y su función: «y corregióle de los antiguos originales que estavan corruptos y mal compuestos en antiguo estilo, por falta de los diferentes y malos escriptores, quitando muchas palabras superfluas y poniendo otras de más polido y elegante estilo tocantes a la cavallería y actos della» (Rodríguez de Montalvo 1987: 225).

El problema de la corrupción textual y el estado incompleto de la obra no solo afecta a la arista didáctica, sino también a la autenticidad del texto. En ese sentido, hay que destacar que el prólogo de Rodríguez de Montalvo presenta a Tito Livio como el gran modelo de la historia: «Otra manera de más convenible crédito tuvo en la su historia aquel grande historiador Titus Livius para ensalçar la honra y fama de los sus romanos» (Rodríguez de Montalvo 1987: 221). Más allá de las consideraciones de contenido, la búsqueda y recuperación de textos latinos y griegos en antiguos manuscritos medievales fue una de las labores centrales del Humanismo durante los siglos XIV a XVI, vínculo ya sugerido por Sarmati (2004), aunque poco desarrollado ${ }^{3}$.

${ }^{3}$ El latín y el griego, además de lenguas sagradas, son las más socorridas en los tópicos del manuscrito encontrado y la falsa traducción y también son aquellas en las que el Humanismo centró principalmente sus labores e intereses (Marín Pina 1994: 545-547). Lo descrito en las obras de Rodríguez de Montalvo y en buena parte de los libros de caballerías aquí estudiados coincide con la situación general del conocimiento de dichas lenguas en la península Ibérica en dicha época y las prácticas de traducción e impresión: «El romanceamiento de textos en lenguas clásicas, griega y latina, o traducción vertical, es una constante significativa durante el período 1500-1680. A lo largo del siglo XvI, a las traducciones de los clásicos (Cicerón, Tácito, Marcial, Epicteto, Aristóteles) se añade el esfuerzo de numerosos humanistas que se vuelven hacia otros textos morales, filosóficos y de espiritualidad y los traducen con el fin de difundirlos de manera efectiva a través de la imprenta. A ellos se suma la traducción de los textos religiosos, empezando por los Evangelios y terminando por la obra erasmista, como parte de un nuevo impulso evangelizador. De manera habitual, el desconocimiento generalizado del griego llevó a emplear textos intermedios latinos o, incluso, en otras lenguas romances. En el caso del latín, las traducciones forman parte de un proceso complejo: permiten incorporar nuevos contenidos; conducen a elevar la lengua castellana hasta el nivel mismo de la lengua de partida; y son un recurso indispensable en la búsqueda del máximo número posible de lectores en el nuevo negocio editorial» (Trujillo 2009: 401-402). 
En particular, el rescate, compilación, corrección y traducción de la obra de Tito Livio, Ab urbe condita libri o las Décadas, que hasta la fecha se encuentra incompleta, fue una de las grandes labores de humanistas como Landolfo Colonna, Francesco Petrarca, Lorenzo Valla o Pero López de Ayala. Petrarca logró compilar la copia más completa de la obra de Livio en muchos siglos:

The manuscript that everyone immediately associates with Petrarch and Avignon is a famous Livy now in the British Library (Harley 2493, Plate $\mathrm{XV}$ ). This volume, which originally contained books 1-10 and 21-40, was put together by Petrarch; parts of it he copied in his own hand. The nucleus of the book is a manuscript of Livy's third decade, written in Italy about 1200 and ultimately derived, as are all the complete manuscripts that have survived, from the extant Puteanus; to this Petrarch added a copy of the first and fourth decades. The various books of Livy's voluminous work, usually in units of ten, had followed their separate fates through the Middle Ages and it was no mean achievement to have put three decades under one cover, especially as the fourth was rare in Petrarch's day; the remaining books of Livy (41-5) were not discovered until the sixteenth century. The text was supplemented, annotated, and corrected by Petrarch himself [...]. (Reynolds/Wilson 1991: 130)

El manuscrito elaborado por Petrarca estuvo más tarde en las manos de Valla, quien también lo corrigió por medio de anotaciones (Reynolds/ Wilson 1991: 131). Las preocupaciones por el estado y la difusión de la obra de Tito Livio llegaron a la Península con la traducción que hizo Pero López de Ayala de la obra a inicios del siglo xv. En su versión, dedicada a Enrique III, se lee:

el qual libro yacia escondido e nunca jamas fue traydo nin leydo en los vuestros reinos que sea traydo agora en publico [...] vos puso en vuestra necesidad de mandar trabajar que este libro trasladase de latyn en francés enla lengua de Castilla porque vos oviesedes enla vuestra corte e en vuestro regno [...]. (Delicado Méndez 1992: 106)

La labor de Petrarca, Valla y López de Ayala respecto a las copias de la obra de Tito Livio son un ejemplo paradigmático de las prácticas más comunes de la cultura escrita de su época y del propio contexto literario de Rodríguez de Montalvo. Tan es así, que la manera en que se plantea el tópico del manuscrito encontrado refleja las prácticas y preocupaciones de la cultura escrita vinculada al Humanismo, ejemplificadas ya con el caso de la obra de Tito Livio: el rescate de textos perdidos, su corrección, autenticidad y restablecer la obra completa. Así, el vínculo del prólogo del Amadís con Livio no es solo por el interés en la definición del género histórico, sino por las prácticas humanistas en torno al rescate textual y la difusión de obras de la Antigüedad grecolatina. Además, la 
práctica de la traducción al castellano a la que remite el Amadís fue uno de los cauces centrales del Humanismo en España en el siglo XV, como muestra el ya citado pasaje de López de Ayala, y de la gran popularidad que tuvo dicha labor para el estamento de Rodríguez de Montalvo: «En España, el movimiento prende entre la nobleza a la moda, que encarga traducciones para acceder a los grandes hechos y doctrinas que encierran las letras antiguas» (Ynduráin 1994: 390).

Tanto el supuesto origen geográfico del texto del Amadís y las Sergas, como su descripción material y su estado de conservación se ligan con referentes extratextuales relevantes de su contexto y las prácticas del Humanismo. En primera instancia, que el manuscrito provenga de una tumba en una ermita cerca de Constantinopla explica que el texto haya permanecido oculto y lo vincula directamente no solo con la geografía del universo amadisiano, como ya se señaló, sino con uno de los eventos más significativos para la Europa del xv: la toma de la ciudad por los otomanos en 1453. Si bien desde el siglo XIV con figuras como Petrarca y Boccaccio se había incrementado el interés por el griego y sus textos en Europa occidental, la caída del Imperio bizantino provocó la llegada de un gran número de refugiados a Italia, quienes trajeron su conocimiento del griego y, en muchas ocasiones, libros en esta lengua. A raíz de esto, ya para 1500 casi toda la literatura de la Grecia Antigua que ha sobrevivido hasta la actualidad se encontraba en bibliotecas europeas, principalmente italianas (Reynolds/Wilson 1991: 146-154; Reeve 1996: 34-37). Luego, la distancia y los lugares recorridos por el manuscrito encontrado permiten presentarlo como un texto exótico cuya llegada a España es parte de los procesos de revaloración del espacio y el poder vinculado a sus lectores y los reinos cristianos del Renacimiento (Campos García Rojas 2012).

Si bien en el prólogo del Amadís no se hace explícita la lengua en la que está escrito el manuscrito, en las Sergas se confirma la suposición de un original en griego a partir de su origen en Constantinopla: «E porque aún tú no has visto ni podido alcançar el fin dello [...] assí como lo fallaron en la tumba de piedra de Constantinopla [...] porque esto está en letra griega, para ti es escusado leerla, pues que la no entenderías, leértelo ha en la tuya esta mi sobrina Julianda» (Rodríguez de Montalvo 2003: 548-549). La llegada del manuscrito greco amadisiano a manos de Rodríguez de Montalvo se presenta de manera muy cercana al fenómeno histórico de la segunda mitad del siglo xv: la llegada a Europa occidental de manuscritos griegos provenientes de Constantinopla. La búsqueda de dichos textos y el interés por el rescate del griego y su literatura corresponden a los intereses y actividades del Humanismo. El traslado del manuscrito encontrado del Amadís y las Sergas y sus detalles, junto con el elogio de la toma de Granada en 1492 con el que abre el prólogo, 
configuran la idea de la translatio imperii y la translatio studii del Imperio bizantino a los reinos de Castilla y Aragón ${ }^{4}$.

Otros aspectos que se describen del manuscrito también apuntan en la misma dirección. En primer lugar, el énfasis paleográfico en la letra griega y las dificultades para leerla, tanto en el prólogo del Amadís como en las Sergas, son otro rasgo de las preocupaciones y quejas del aprendizaje textual del Humanismo español: «El mismo afán de claridad caligráfica [para el griego] propugnado por Nebrija fue sustentado también por Juan Luis Vives [...]. La "buena letra", según expresión frecuente y bien documentada en la época, fue una pretensión estética de honda raigambre humanística [...]» (Escobar 2020: 198-199)

Por otra parte, en los pasajes donde se describen las supuestas crónicas se hace referencia al soporte, pergamino antiguo, y el pobre estado de conservación, con la consecuente dificultad de lectura para aquellos entendidos en su lengua. Además de las letras, las otras características materiales del manuscrito refuerzan el nexo con las labores de rescate de libros antiguos del Humanismo. El material de este supuesto manuscrito estaría en claro contraste con un soporte relativamente nuevo que comenzó a abundar más y más en Europa, desde el siglo XIII: el papel, soporte en el que circularía el Amadís de Rodríguez de Montalvo por todo el continente. Junto con su estado de conservación, la breve descripción del soporte permite enfatizar que se trata de un texto auténtico y de la Antigüedad. Así, el pergamino no solo proviene del lugar donde sucede el clímax de la trama, sino que sus rasgos hacen suponer que proviene de esa remota época en la que sucedieron los hechos de Amadís y Esplandián: «No muchos años después de la passión de nuestro Redemptor y Salvador Jesuchristo [...]» (Rodríguez de Montalvo 1987: 227).

Las continuaciones del ciclo de Amadís de Gaula, posteriores a Rodríguez de Montalvo, se ciñeron a lo planteado en las obras del autor medinés. La rama heterodoxa del ciclo, el Florisando (1510) de Páez de Ribera y el Lisuarte de Grecia (1526) de Juan Díaz, reconocieron los

${ }^{4}$ Compárese el caso de los libros de caballerías de Rodríguez de Montalvo con el caso medieval del Libro del cavallero Zifar: «E porque la memoria del ome ha luengo tiempo, e no se pueden acordar los omes de las cosas mucho antiguas sy las non fallan por escripto, e porende el trasladador de la estoria que adelante oyredes, que fue trasladado de caldeo en latin e de latin en romançe, puso e ordeno estas dos cosas sobredichas en esta obra, porque los que venieren después de los deste tiempo, sera quando el año jubileo a de ser, porque puedan yr a ganar los bienauenturados perdones que en aquel tiempo son otorgados a todos los que alla fueren, e que sepan que este fue el primer cardenal que fue enterrado en España. Pero esta obra es hecha so emienda de aquellos que la quesieren emendar. E çertas deuenlo fazer los que quisieren e la sopieren emendar sy quier; porque dize la escriptura: "Qui sotilmente la cosa fecha emienda, mas de loar es que el que primeramente la fallo"» (Wagner 1929: 6). El Zifar vincula la traducción de la supuesta fuente con una lección de índole explícitamente religiosa y no da detalle textual o material alguno salvo las lenguas por las que pasó el texto.

${ }^{5}$ El aprendizaje del griego, tanto en su dimensión oral como escrita, es un tema importante en las biografías de Amadís y Esplandían (Campos García Rojas 2005; Escobar 2020: 196). 
referentes humanistas planteados en el Amadís y las Sergas, recurrieron a estos nuevamente e, inclusive, los acentuaron al hacer explícitos los vínculos con Petrarca. El Florisando alude a un supuesto texto en griego, que pasó por a la biblioteca florentina de Petrarca: «Firalites, discípulo que fue del gran Petrarca, después de la muerte de su maestro havía hallado en su librería un libro escripto en lengua griega, e él Firalites lo sacó en lengua toscana» (Páez de Ribera 2018: 22). Como en las obras de Rodríguez de Montalvo, el Florisando plantea, gracias al libro de la biblioteca petrarquista, no solo continuar la obra, sino corregir la obra previa. En particular, el Florisando modificó el encantamiento narrado al final de las Sergas y depuró a la familia amadisiana de la magia, las doncellas errantes y las aventuras de la caballería errante, acorde con la ideología del texto y con las quejas de diversos humanistas sobre el contenido poco provechoso de los libros de caballerías para la edificación cristiana de sus lectores.

Si bien no hay más información sobre el libro de Firalites y no se dan detalles de su aspecto y materialidad, su autenticidad y antigüedad no solo están dados por la lengua de origen, el griego, sino por su tránsito por la biblioteca de Petrarca y las famosas labores de rescate textual emprendidas por él, quien funciona como auctoritas del rescate de textos griegos. La referencia al toscano remite a las prácticas comunes del Humanismo castellano del siglo $\mathrm{XV}$, donde dicha lengua fue un puente importante entre los autores clásicos y su difusión castellana:

The Castilian translations of Greek works by Procopius, Basil, Plato, Lucian, Homer, Aristotle, Plutarch, Hermes Trismegistus, and Appian were made from Latin or Italian intermediary versions, adaptations, and falsifications by the humanists Leonardo Bruni, Cencio de Rustici, Pier Candido Decembrio, Giovanni Aurispa, Marsilio Ficino, and others. Kristeller has noted the striking propensity of Santillana and other Spanish patrons to commission Tuscan translations of the classics. (Lawrance 2014: 222)

Así, el Florisando, como otros libros de caballerías que se señalarán adelante, recrea los detalles de los textos que circulaban en la época, sus procesos de difusión y sus características establecidos por el Humanismo.

La continuación directa del Florisando, el Lisuarte de Grecia de Juan Díaz, retoma elementos de las obras de Páez de Ribera y Rodríguez de Montalvo para explicar su origen textual:

Y esta es la mera verdad de la historia y por ser mas firme della vi la historia y original que es la propia de los emperadores de Constantinopla, porque quando por nuestros pecados aquel gran Imperio de Constantinopla se perdió y fue ganado de los turcos, el coronista mayor del Emperador fuyó con las corónicas antiguas, viejas y nuevas y se acogió ala isla de Rodas 
y allí moró algunos días y toda la librería dexó al maestre de la Orden de Sant Juan rogando le muy afincadamente que la hiziesse guardar como cosa de tanto valor que aunque el señorío se perdisse que las famosas vidas de aquellos que lo ganaron no fuesen perdidas ni trasntornadas en el olvido de las gentes el qual coronista que fuera del Emperador, como fuesse natural de Florencia, traxo esta historia escripta de su mano en lengua toscana, porque en estas partes oviesse memoria de la qual corónica y historia en toscano fue sacada esta grande historia sin faltar ni acrecentar palabra la qual en la misma guisa avia sido trasladada del original griego [...]. (Díaz 1526: ff. 100rb-100va)

En primer lugar, aparece la llegada de una crónica tras la caída de Constantinopla, como se planteaba de manera implícita en el Amadís, aunque, en este caso, es gracias a una figura de prestigio como el cronista imperial de la ciudad. Sin duda, la especial atención que pone el pasaje a la conservación de la crónica enfatiza el valor de la obra de Díaz, al punto de que en este relato se involucra explícitamente a una orden de caballería sobreviviente de las cruzadas, cuyo centro se encontraba en la isla de Rodas, la orden de San Juan de Jerusalén, también conocida como los Caballeros Hospitalarios. Esto suma una segunda peripecia a la supervivencia de la supuesta crónica, pues en 1522, cuatro años antes de la publicación del Lisuarte de Juan Díaz, los Hospitalarios abandonaron Rodas derrotados, tras un sitio de meses liderado por Solimán, y permanecieron ocho años deambulando por Europa hasta establecer su nuevo centro de operaciones en Malta (Abela 2018: 1-2). Tras referir a ambas derrotas de la cristiandad, se destaca la importancia de la supervivencia de la crónica por encima de la de los señoríos, pues en esta se guardan los ejemplos de una genealogía de caballeros cristianos triunfantes. Así, al detallar el viaje, las vicisitudes y los cuidados para conservar la crónica, con base en referentes extratextuales de los lectores del XVI y de manera más amplia que en el Amadís de Gaula, se refuerza no solo la autenticidad, sino la importancia de la crónica. La historia del rescate justifica la necesidad de difundir una historia antigua, así como completar y ampliar las hazañas de la familia amadisiana.

En segunda instancia, la historia de la supuesta crónica original del Lisuarte de Díaz retoma el nexo explícito con las labores humanistas siguiendo los referentes del Florisando. Si bien el Lisuarte no menciona directamente a Petrarca, la caracterización del cronista como florentino lo relaciona con este personaje ya mencionado en la obra de Páez de Ribera, en especial por haber elaborado la crónica en toscano, lengua de la cual supuestamente se tradujo la obra al castellano, como en el caso del Florisando. De igual manera, en el Lisuarte se omiten detalles concretos de su estado de conservación y materialidad.

Cabe señalar que en este caso los tópicos del manuscrito encontrado y la falsa traducción no aparecen en los paratextos o en el inicio 
del texto, sino a la mitad. Su posición está relacionada con otro de los aspectos humanistas ya resaltados por el Amadís y el Florisando: la corrección del texto antiguo y corrupto por su transmisión. En este caso, la historia del viaje de Constantinopla introduce en la obra testimonios de otros viajeros para hacer una corrección simple sobre un personaje secundario, don Guilán, quien había muerto en la defensa de Constantinopla en las Sergas, pero que había reaparecido con vida en el Florisando. El Lisuarte reafirma la muerte del personaje, realizando una corrección menor a la trama de la obra de Páez de Ribera (Gutiérrez Trápaga 2015: 511-512). Entonces, aparece en el relato no solo el rescate y la difusión de una historia antigua, sino su corrección, como en los libros previos del ciclo amadisiano.

Esta rama del ciclo amadisiano, la heterodoxa, retoma los tópicos del manuscrito encontrado y la falsa traducción a partir de variaciones de los elementos planteados en el Amadís de Gaula sobre su origen, materialidad y estado de conservación. La reaparición de estos elementos en el Florisando y el Lisuarte de Grecia de Juan Díaz permite relacionarlo también con las prácticas del Humanismo en torno al rescate, corrección, traducción y difusión de los textos. Inclusive, el Florisando es aún más explícito al plantear directamente la biblioteca de Petrarca como uno de los estados en la transmisión y conservación del texto.

Por su parte, la otra rama de continuaciones del ciclo amadisiano, compuesta casi en su totalidad por obras de Feliciano de Silva, también retomó estos tópicos en donde se describen las supuestas crónicas. El Lisuarte de Grecia (1514) de Feliciano de Silva lo hace de manera tímida en comparación a sus posteriores continuaciones: «acordé la presente crónica del famosíssimo cavallero Lisuarte de Grecia, que nuevamente fue hallada en Londres, de emendalla de algunos vocablos que por la mucha antigüedad estavan corruptos» (Silva 2002: 4). Este ejemplo no establece una relación geográfica clara con los textos de la antigüedad grecorromana planteada por el Humanismo, en lugar de eso apela a Londres, centro geográfico de origen de la familia amadisiana. De cualquier manera, permanece la idea del rescate textual para completar la historia y la necesidad de restaurarlo a su estado original.

La siguiente obra de Feliciano de Silva, el Amadís de Grecia (1530), amplió la narración en torno a sus orígenes textuales ficticios:

la hallará en una cueva que se llama los Palacios de Hércules, metida en una caxa de madera que no se corrompe, en un lado de la pared, porque cuando España fue perdida la escondieron en aquel lugar porque la memoria d'estos cavalleros no se perdiesse. Y hallarla as en lengua latina, la cual de griego fue sacada, y con la antigüedad algo corrompido el estilo. (Silva 2004: 246-247) 
Si bien en el pasaje no se refiere a la supervivencia del manuscrito en la caída de Constantinopla, sí aparece la pérdida de otro reino cristiano, en este caso el visigodo de Toledo en el año 711. Esto permite caracterizar la antigüedad y la importancia del texto, así como explicar que no fuera conocido previamente y el lugar de su hallazgo, rasgo que según Delpech (1998: 10-17) le otorga propiedades mágicas implícitas.

Como en su obra previa, en el Amadís de Grecia no hay una relación explícita con Constantinopla o Petrarca, pero sí se especifican las lenguas: el latín a partir de un original griego. El texto griego traducido al latín se repite en otras de sus continuaciones, por ejemplo el Florisel de Niquea I-II (1532): «Crónica [...] emendada del estilo antiguo según que la escrivió Zirfea, reina de Argines, por el grande amor que a sus padres tuvo, que fue traduzida de griego en latín y de latín en romance castellano» (Silva 2015: 13). En la siguiente continuación, el Florisel de Niquea III (1535), aparece lo siguiente: «La cual fue corregida por Feliciano de Silva de algunos errores que en la traslación que se hizo del griego en latín por el gran historiador Falistes Campaneo avía» (Silva 1999: 3). Estas dos lenguas se convirtieron en una constante a imitación de lo planteado por Rodríguez de Montalvo en la línea de la imitación de los intereses y labores de los humanistas. Claro está, en el último ejemplo de Feliciano de Silva, hay que destacar la burla implícita en el nombre del traductor que le otorga un matiz irónico, como apunta Coduras: «Nombre de procedencia latina. De falir "faltar uno a su palabra y crédito" (Covarrubias 2006: 879), y constituiría un juego más de Feliciano de Silva al cuestionar la verosimilitud e integridad de uno de los transmisores ficticios de su historia» (Coduras Bruna s/f).

A comparación de lo narrado en las obras de Rodríguez de Montalvo, Páez de Ribera y Juan Díaz, Feliciano de Silva ofrece menos detalles sobre el estado material y la historia de la transmisión de las crónicas supuestamente encontradas y traducidas. De manera muy sintética señala los idiomas por los que pasó el texto, la necesidad de corregir y completar la historia, así como la antigüedad. Destaca el ya señalado caso del Florisel de Niquea III, pues la burla a los supuestos traductores latinos indica que el manuscrito y la falsa traducción eran tópicos del género, al igual que el vínculo con las labores del Humanismo. Así, estos tres aspectos estaban suficientemente consolidados en el horizonte de expectativas del género para que apareciera una burla a la transmisión ficticia de los textos caballerescos a imitación del Humanismo.

\section{OTROS LIBROS DE CABALLERÍAS}

Fuera del ciclo de Amadís de Gaula, diversos libros de caballerías retomaron con algunas variaciones los elementos que configuran la descripción física y la historia de los libros de crónicas en los que 
supuestamente se basaron las correcciones y añadiduras de Rodríguez de Montalvo. Por ejemplo, el Cristalián (1545) de Beatriz Bernal refiere su origen a un libro tomado de un sepulcro de una iglesia a la manera del Amadis de Gaula:

[...] yendo un viernes de la Cruz con otras dueñas a andar las estaciones (ya que la aurora traya el mensaje del venidero día) llegamos a una yglesia, a donde estava un muy antiguo sepulchro, en el qual vimos estar un defuncto embalsamado: y yo siendo mas curiosa que las que comigo yvan, de ver y saber aquella antigüedad, llegueme más cerca: y mirando todo lo que en el sepulchro avía, vi que a los pies del sepultado estava un libro de crecido volumen. (Bernal 2015: 891)

Destaca en este pasaje el tamaño y la antigüedad del libro encontrado, cuyos detalles se amplían en la descripción de la labor de la autora:

el qual (aunque fuesse sacrilegio) para mi aplique: y acuciosa de saber sus secretos, dexada la compañía, me vine a mi casa: y abriéndole, halle que estava escripto en nuestro común lenguaje, de letra tan antigua, que ni parecía Española, ni Araviga, ni Griega: Pero toda via, creçiendo mi desseo, y abraçandome con un poco de trabajo, vi en el muy diversas cosas escriptas: de las quales, como pude, traduxe y saque esta historia. (Bernal 2015: 891)

Este pasaje gira en torno a la misteriosa letra antigua que dificulta identificar la lengua. Si bien el idioma resulta ser español, la labor paleográfica con el texto se presenta como una traducción en la medida en que el texto requiere ser trasladado de la vieja letra a una nueva para dar a conocer la historia. Geográficamente, el origen del texto es la propia España y no Constantinopla, aunque en este caso no se alude a la caída o pérdida de ningún reino cristiano. El lugar de origen del texto es el mismo que el del caballero protagonista, Cristalián de España, planteando, como en otros libros de caballerías, continuidad entre el espacio de la ficción y el de los lectores del siglo XVI.

Por su parte, el prólogo del Felixmarte de Hircania (1556) de Melchor Ortega ofrece un largo relato sobre su supuesto manuscrito fuente, que desde un inicio se presenta como resultado de una búsqueda intencional por textos antiguos, tanto en español como en toscano: «Soy tan inclinado a lición antigua de historia verdadera que esto me ha hecho ser curioso en buscar libros antiguos, especial en nuestra lengua y la toscana; y si los hallo en otra que no entienda, procuro quién me los declare en qualquier parte que esté» (Ortega 1998: 11). A continuación, el relato precisa el lugar y la fecha del descubrimiento, vinculando el Felixmarte a uno de los grandes proyectos de la bibliofilia humanista en España de inicios del siglo XVI, la Biblioteca Colombina: 
Y entre las otras que me he hallado - con este cuidado y diligencia- fue el año de treinta y nueve en la ciudad de Sevilla, donde siendo avisado de la gran copia de libros de diversas lenguas que en el monesterio de Sant Pablo dexó un hermano de don Christoval Colón -primero descubridor de la isla Española-, gasté muchos días en ver y leer alguna parte dellos, y entre éstos el original del nuestro en lengua toscana. (Ortega 1998: 11)

El año de 1539, referido en el pasaje, fue el del fallecimiento de Hernando de Colón, principal responsable de la biblioteca e hijo, que no hermano, de Cristóbal Colón. No se recurre a la caída de Constantinopla o el reino visigodo para explicar la aparición del texto, sino a una biblioteca humanista y sus labores de recuperación y recopilación de libros, hecho que desplaza los hallazgos en sepulcros y misteriosas cuevas de otros relatos en el género.

El prólogo continúa resaltando el estado de deterioro del manuscrito y la consecuente dificultad para rescatar el texto. Además, se atribuye la traducción del latín al toscano a Petrarca, ampliando la intervención del Humanismo en el proceso de rescate de la obra:

tan antigua que con gran trabajo se podía leer, porque en el tiempo avía gastado y consumido mucha parte de la scriptura, que por razón se avía de entender, y juntóse con esto los vocablos no usados en este tiempo, aunque de la verdadera y propia lengua toscana, porque el que de lengua latina en ella lo traduxo fue el excelente poeta y orador Francisco Petrarca, florentino, y en su traducción nos muestra averlo sido de griego en lengua latina, por el gran Plutarco, discípulo de aquel famoso historiador Philostio Atheniense, que en lengua griega esta gran historia escrivió por mandado del gran senado de Athenas, en el qual padre de la eloquencia y verdad fue llamado. (Ortega 1998: 11-12).

Por medio de Plutarco, el pasaje establece la filiación del texto a la historia y a la lengua griega. Así, se presenta la versión castellana de la crónica, el propio Felixmarte, como el último eslabón de transmisión de la literatura griega, gracias a los esfuerzos filológicos del Humanismo tanto florentino como español. Como en el caso de la mención a la lengua toscana y a Petrarca, la presencia de Plutarco como parte de la transmisión de la obra refleja uno de los intereses contemporáneos reales y más importantes del Humanismo, tanto en Italia como en España de los siglos XV y XVI (Morales Ortiz 2000: 75-94; Pade 2014: 537-542; Pérez Jiménez 2014: 558-573; 2019: 607-613). Así, todos los elementos del manuscrito encontrado y la falsa traducción buscan otorgarle credenciales humanistas al Felixmarte.

El Espejo de príncipes y caballeros (1555) de Diego Ortúñez de Calahorra también se presenta como una traducción de un texto griego: 
[...] según que Artimidoro el griego en los grandes volúmenes de sus corónicas lo dexó escripto [...] Y porque en los venideros tiempos tan altas cosas no fuessen perdidas de memoria, muchos de los griegos las recopilaron, y dellas hizieron doze libros, el primero de los quales es éste que se sigue, que es el principio de todas las historias de Trebacio. Parece por una corónica, de las más auténticas y antiguas de los griegos. (Ortúñez de Calahorra 1975: 26 y 30-31)

El pasaje remite a un original griego atribuido a un autor con referente extratextual real, como Artemidoro. Además, la descripción pone énfasis en el tamaño de las crónicas. Tal detalle concuerda con la extensión del Espejo y la posibilidad de establecer un ciclo compuesto de varias obras, tal y como sucedió, si bien no se llegaron a escribir las doce partes como sí lo logro el ciclo amadisiano (Campos García Rojas 2008; Ramos Nogales 2016). Más allá de ese detalle, el planteamiento del manuscrito encontrado y la falsa traducción en el texto de Ortúñez de Calahorra implica difundir y buscar completar una historia griega perdida.

En esa misma línea, el texto compara explícitamente la supuesta difusión de la historia del Espejo con la de la Odisea y el corpus homérico, que se dice llegó a superar:

Y ni la Ulixea de Homero, ni ninguno de sus cantos e sonorosos versos por largos tiempos en toda Grecia no se oyó cantar después que los hechos destos cavalleros a los griegos fueron manifiestos. Déstos hazían muy largos cuentos, e con mil maneras de versos cantavan sus amores, e no se hazía edificio de casa ni pintura que alguna historia dellos o de sus espantosos hechos no llevasse. De manera que por ninguna parte de toda Grecia se podía passar que no se contassen o cantassen o estuvieran pintadas las historias y muy maravillosos hechos destos cavalleros, como que ninguna otra cosa que de armas o de amores fuese paresciese serles agradable. (Ortúñez de Calahorra 1975: 29-30)

Este detallado relato de la difusión de las historias del Espejo hiperboliza la importancia de la obra, al presentarla como superior a la literatura homérica, y recurrir a las coordenadas de los intereses y las prácticas textuales del Humanismo.

Por último, en el caso de un libro de caballerías manuscrito, Mexiano de la Esperança (1583) de Miguel Daza, aparecen varios de los elementos señalados, aunque con un matiz irónico, como sugiere su editora moderna (Martínez Muñoz 2019: XII). En dicha obra, Nictemeno, el cronista, afirma haber encontrado el primer libro de caballerías jamás escrito:

Con esto, fui y le truxe la istoria o aventuras de Cleántulo, con los amores de Sineo y Polidea, que entiendo que fue el primer libro que llamáis de matahombres o de caballerías del mundo. Porque os digo verdad cierto cierto, a fe de bueno, que yo le allé de mano en pergamino 
en un lugarexo pequeño del reino de Castilla, en poder de un herrero. El lugar se llama Pozancos y él se llamaba Ulano García, hombre de más de setenta y cinco años (perdóneme el señor herrero), que era morisco del reino de Aragón y decía que de África le havía traído su agüelo que fue alfaquí según él decía. Él estaba en latín y muy rebueno, el título era este, cierto buelto en español: Libro de los heroicos echos del príncipe griego Cleántulo, con los estremados amores de Sineo y Polidea, compuesto por Aristófanes trágico, traducido en latín por Quintilo Cremonense. Esto es verdad cierto, si era fabuloso el título yo no lo sé, lo que sé es que era estremado latín y que nada debía a Quintiliano ni a Cicerón. (Daza 2019: 502-503)

Si bien el pasaje remite a viejos pergaminos, la persona, el lugar y la geografía del hallazgo contrastan con la tradición de lugares vinculados a la caída de imperios cristianos, tumbas, cuevas o prestigiosas bibliotecas, mostrando un aspecto modesto y con tono burlesco, respecto al horizonte de expectativas del género. Nuevamente, se remite a un original griego, traducido al latín. La mención a Aristófanes, autor de tragedias y no de libros de caballerías, junto con el nombre del traductor latino y el comentario al respecto, refuerzan la burla de los tópicos a imitación del Humanismo en el género.

\section{ConClusión}

Los casos aquí presentados muestran cómo algunos de los libros de caballerías establecieron nexos explícitos con el Humanismo y sus labores en los detalles de los tópicos del manuscrito encontrado y la falsa traducción, aún en los casos que ya introducen ironía o elementos cómicos. En los libros examinados, hay abundantes menciones a Petrarca y sus bibliotecas, así como a los principales autores trabajados por el toscano y sus seguidores posteriores, a saber, Tito Livio, Plutarco y Homero. Además, se refleja la realidad de los trabajos emprendidos por el Humanismo: desde la búsqueda y rescate de los antiguos manuscritos griegos y latinos, así como su paso por diversas lenguas, en particular el toscano, antes de ser llevados al castellano. La nómina de autores vinculados a dichos procesos, como Homero, Plutarco, Tito Livio o Petrarca, refuerzan de manera clara el mismo espíritu.

Tras los tópicos del manuscrito encontrado y la falsa traducción, aparecen los detalles de la supervivencia y la transmisión de los supuestos manuscritos fuentes del relato. Las narraciones y descripciones en estos episodios también contribuyen al retrato de las labores del Humanismo previo y contemporáneo a los libros de caballerías: los viejos, maltratados y corruptos manuscritos permiten poner énfasis en la necesidad de corregir y completar dichos textos. Además, la labor de rescate textual remite frecuentemente a Constantinopla, ciudad que tuvo un papel crucial para 
el rescate del griego y su literatura en Europa Occidental, muchas veces mediada por las traducciones latinas. Continuamente, aparece el rescate de textos perdidos, tanto de geografías remotas, bibliotecas y tumbas.

Desde la perspectiva de la materialidad de los textos y sus procesos históricos al interior de la ficción, los manuscritos en los libros de caballerías aquí estudiados se muestran como el resultado de las pesquisas del Humanismo. Destaca la presencia y centralidad del manuscrito a lo largo de un género que fue pilar de la imprenta europea y que circuló principalmente en forma de impresos. El manuscrito y sus rasgos e historia material representan el prestigio de lo antiguo, aunque el contenido se transmita ahora de manera impresa, específicamente vinculan al género con la tradición grecolatina. En ese sentido hay que señalar que dicho vínculo no se limita a la presentación de la materialidad de las fuentes, pues los libros de caballerías recurren en diversos episodios a una serie de elementos de la literatura grecolatina: personajes, descripciones, lugares y tópicos.

Queda, pues, por realizar un amplio estudio del género que revise la relación del Humanismo y la tradición grecolatina con las distintas poéticas de los libros de caballerías y se plantee una relación más compleja entre ambos de lo que se ha hecho hasta ahora, como lo sugieren los manuscritos al interior de la ficción. Igualmente, dada la diversidad literaria del género, habría que examinar con mayor profundidad, ya en otros episodios, ya en otros libros de caballerías, la presencia y rasgos de distintos manuscritos que no establecen vínculos explícitos con el Humanismo y sus labores, como sucede con la presencia de los grimorios. Así, por medio del estudio de la representación de la materialidad en la ficción, este trabajo mostró que una parte importante de los libros de caballerías concibe el universo literario en términos predominantemente manuscritos y de manera muy similar al de los humanistas contemporáneos.

\section{REFERENCIAS BIBLIOGRÁFICAS}

ABELA, Joan (2018), Hospitaller Malta and the Mediterranean Economy in the Sixteenth Century. Woodbridge: Boydell.

Bernal, Beatriz (2015), Cristalián de España. Jodi Growitz (ed.). Newark: Juan de la Cuesta.

Bognolo, Anna (1998), «El prólogo del Amadís de Montalvo entre retórica, poética e historiografía», en María Cruz García de Enterría y Alicia Cordón Mesa (eds.), Actas del IV Congreso Internacional de la Asociación Internacional Siglo de Oro (AISO), (Alcalá de Henares, 22-27 de julio de 1996). Alcalá de Henares: servicio de Publicaciones de la Universidad de Alcalá, vol. 1, pp. 275-281.

Campos García Rojas, Axayácatl (2005), «Las lenguas extranjeras en los libros de caballerías: Amadís de Gaula y Las Sergas de 
Esplandián», en Josep Lluís Martos, Josep Miquel Manzanaro i Blasco y Rafael Alemany Ferrer (eds.), Actes del X Congrés Internacional de l'Associació Hispànica de Literatura Medieval: Alicante, 16-20 de septiembre de 2003. Alicante: Institut Interuniversitari de Filologia Valenciana, vol. 1, pp. 487-497.

CAmpos García Rojas, Axayácatl (2008), «El Espejo de príncipes y caballeros: su ciclo y el final de un género», en Aurelio González y María Teresa Miaja de la Peña (eds.), Caballeros y libros de caballerías. México: Universidad Nacional Autónoma de México, Facultad de Filosofía y Letras, pp. 163-182.

CAMpos García RoJas, Axayácatl (2012), «Variaciones en centro y periferia sobre el manuscrito encontrado y la falsa traducción en los libros de caballerías castellanos», Tirant: Butlletí informatiu i bibliogràfic de literatura de cavalleries, 15, pp. 47-60. En línea: $<$ http://parnaseo. uv.es/Tirant/Butlleti.15/4Art_Campos.pdf> [consulta: 29/09/2020].

Coduras Bruna, María (s. f.), «Falistes Campaneo», DINAM. Diccionario de nombres del ciclo amadisiano. Zaragoza: Universidad de Zaragoza. En línea: <http://dinam.unizar.es/ver/id/482.html> [consulta: $28 / 08 / 2020]$.

Covarrubias Horozco, Sebastián de (2006), Tesoro de la lengua castellana o española. Ignacio Arellano y Rafael Zafra (eds.). Madrid/ Frankfurt am Main: Universidad de Navarra/Iberoamericana/Vervuert/Real Academia Española y Centro para la Edición de los Clásicos Españoles.

DaviES, Martin (1996), «Humanism in Script and Print in the Fifteenth Century», en Jill Kraye (ed.), The Cambridge Companion to Renaissance Humanism. Cambridge: Cambridge University Press, pp. 47-62.

DazA, Miguel (2019), Corónica de don Mexiano de la Esperança, Caballero de la Fe. Ana Martínez Muñoz (ed.). Alcalá de Henares: Servicio de Publicaciones de la Universidad de Alcalá.

Delicado Méndez, Rosario (1992), «La tradición directa de Tito Livio en castellano», Helmántica, 43/130-132, pp. 105-110. En línea: $<$ https://summa.upsa.es/viewer.vm?id=3334\&view=main\&lang=es $>$ [consulta: 15/09/2020].

DelPECH, François (1998), «El hallazgo del escrito oculto en la literatura española del Siglo de Oro: elementos para una mitología del libro», Revista de Dialectología y Tradiciones Populares, 53/1, pp. 5-38.

Demattè, Claudia (2005), «La mise en abyme en los libros de caballerías hispánicos», en Mercedes Pampín Barral y Carmen Parrilla García (eds.), Actas del IX Congreso Internacional de la Asociación Hispánica de Literatura Medieval: (A Coruña, 18-22 de septiembre de 2001). La Coruña: Universidade da Coruña, Departamento de Filoloxía Española e Latina/Toxosoutos, vol. 2, pp. 189-204.

DíAz, Juan (1526), Lisuarte de Grecia. Sevilla: Juan y Jacobo Cromberger. 
Enciso Zarate, Francisco de (2009), Florambel de Lucea (Primera parte, Libros I-III). María del Rosario Aguilar Perdomo (ed.). Alcalá de Henares: Centro de Estudios Cervantinos.

EISENBERG, Daniel (1974), «The Pseudo-Historicity of the Romances of Chivalry», Quaderni Ibero-Americani, 45-46, pp. 253-259.

EsCOBAR, Ángel (2020), «El aprendizaje de la escritura griega por parte de los humanistas españoles», en Christian Brockmann, Daniel Deckers, Dieter Harlfinger y Stefano Valente (eds.), Griechisch-byzantinische Handschriftenforschung: Traditionen, Entwicklungen, neue Wege. Berlin: De Gruyter, vol. 1, pp. 195-200.

Fogelquist, James Donald (1982), El Amadís y el género de la historia fingida. Madrid: José Porrúa Turanzas.

García Gual, Carlos (1996), «Un truco de la ficción histórica: el manuscrito reencontrado», 1616: Anuario de la Sociedad Española de Literatura General y Comparada, 10, pp. 47-60.

GutiÉRREZ TrÁPAGA, Daniel (2015), «Continuar y reescribir: el manuscrito encontrado y la falsa traducción en las continuaciones heterodoxas del Amadís de Gaula», en Marta Haro Cortés (ed.), Literatura y ficción: «estorias», aventuras y poesía en la Edad Media. Valencia: Universitat de València, vol. 2, pp. 503-518.

GutiÉRREZ TrÁPAGA, Daniel (2017), «Battling Narratives in the Amadís Cycle: The Case of Florisando and Sergas de Esplandián», Bulletin of Hispanic Studies, 94/1, pp. 19-34. DOI: https://doi.org/10.3828/ bhs.2017.2.

Lawrance, Jeremy N. H. (2014), «Humanism in the Iberian Peninsula», en Anthony Goodman y Angus Mackay (eds.), The Impact of Humanism on Western Europe During the Renaissance. New York: Routledge.

ManN, Nicholas (1996), «The origins of humanism», en Jill Kraye (ed.), The Cambridge Companion to Renaissance Humanism. Cambridge: Cambridge University Press, pp. 1-19.

Marín Pina, María Carmen (1994), «El tópico de la falsa traducción en los libros de caballerías españoles», en María Isabel Toro Pascua (ed.), Actas del III Congreso de la Asociación Hispánica de Literatura Medieval (Salamanca, 3 al 6 de octubre de 1989). Salamanca: Biblioteca Española del Siglo XV/Departamento de Literatura Española e Hispanoamericana, vol. 1, pp. 541-548.

Marín Pina, María Carmen (ed.) (1998), Primaleón. Alcalá de Henares: Centro de Estudios Cervantinos.

Martínez MuÑoz, Ana (2019), «Introducción», en Miguel Daza, Corónica de don Mexiano de la Esperança, Caballero de la Fe. Ana Martínez Muñoz (ed.). Alcalá de Henares: Servicio de Publicaciones de la Universidad de Alcalá, pp. IX-LIv. 
McKenzie, Donald F. (2004), Bibliography and the Sociology of Texts. Cambridge: Cambridge University Press.

Morales Ortiz, Alicia (2000), Plutarco en España: traducciones de Moralia en el siglo XVI. Murcia: Universidad de Murcia.

OrTEGa, Melchor de (1998), Felixmarte de Hircania. M. ${ }^{a}$ Rosario Aguilar Perdomo (ed.). Alcalá de Henares: Centro de Estudios Cervantinos.

OrtúÑez de Calahorra, Diego (1975), Espejo de príncipes y caballeros. Daniel Eisenberg (ed). Madrid: Espasa-Calpe, vol. 1.

PADE, Marianne (2014), «The Reception of Plutarch From Antiquity to the Italian Renaissance», en Mark Beck (ed.), A Companion to Plutarch. Chichester: Wiley Blackwell, pp. 531-543.

PAÉz DE Ribera (2018), Florisando. M. ${ }^{a}$ Aurora García Ruiz (ed.). Alcalá de Henares: Universidad de Alcalá/Universidad de Jaén.

PÉREZ JiMÉNEZ, Aurelio (2014), «The Reception of Plutarch in Spain», en Mark Beck (ed.), A Companion to Plutarch. Chichester: Wiley Blackwell, pp. 556-576.

PÉREZ JiMÉNEZ, Aurelio (2019), «Plutarch's Fortune in Spain», en Sophia Xenophontos y Katerina Oikonomopoulou (eds.), Brill's Companion to the Reception of Plutarch. Leiden/Boston: Brill, pp. 606-621.

Ramos Nogales, Rafael (2016), «Dos nuevas continuaciones para el Espejo de príncipes y caballeros», Historias Fingidas, 4, pp. 41-95. DOI: 10.13136/2284-2667/50.

ReEve, Michael D. (1996), «Classical scholarship», en Jill Kraye (ed.), The Cambridge Companion to Renaissance Humanism. Cambridge: Cambridge University Press, pp. 20-46.

Reynolds, Leighton Durham y Nigel Guy WiLson (1991), Scribes and Scholars: A Guide to the Transmission of Greek and Latin Literature. Oxford: Oxford University Press.

RodríGuEZ De Montalvo, Garci (1987). Amadís de Gaula. Juan Manuel Cacho Blecua (ed.). Madrid: Cátedra, 2 vols.

Rodríguez de Montalvo, Garci (2003), Sergas de Esplandián. Carlos Sainz de la Maza (ed.). Madrid: Castalia.

SARMATI, Elisabetta (1996), Le critiche ai libri di cavalleria nel Cinquecento spagnolo: con uno sguardo sul Seicento: un'analisi testuale. Pisa: Giardini.

SARMATI, Elisabetta (2004), «Le fatiche dell'Umanista: il manoscritto ritrovato nei libri di cavalleria e nel Don Quijote. Qualche riflessione ancora sul motivo della falsa traduzione», en Folke Gernert (ed.), Letteratura cavalleresca tra Italia e Spagna (Da Orlando al Quijote). Literatura caballeresca entre España e Italia (Del Orlando al Quijote). Salamanca: Seminario de Estudios Medievales y Renacentistas, Sociedad de Estudios Medievales y Renacentistas/CERES de la Universidad de Kiel, pp. 372-392. 
SiLVA, Feliciano de (1999), Florisel de Niquea. Tercera parte. Javier Martín Lalanda (ed.). Alcalá de Henares: Centro de Estudios Cervantinos.

SiLVA, Feliciano de (2002), Lisuarte de Grecia. Emilio José Sales Dasí (ed.). Alcalá de Henares: Centro de Estudios Cervantinos.

SiLva, Feliciano de (2004), Amadís de Grecia. Ana Carmen Bueno Serrano y Carmen Laspuertas Sarvisé (eds.). Alcalá de Henares: Centro de Estudios Cervantinos.

SiLVA, Feliciano de (2015), Florisel de Niquea (Partes I-II). Linda Pellegrino (ed.). Alcalá de Henares: Universidad de Alcalá.

Trujillo, José Ramón (2009), «La edición de traducciones medievales en la Edad de Oro. Textos e impresos de la materia artúrica hispánicas», Edad de Oro, 28, pp. 401-448.

Wagner, Charles Philip (ed.) (1929), El Libro del Cauallero Zifar: (El Libro del Cauallero de Dios). I. Text edited from the three extant versions. Ann Arbor: University of Michigan.

YNDURÁrN, Domingo (1994), Humanismo y Renacimiento en España. Madrid: Cátedra.

Recibido: $1 / 11 / 2020$

Aceptado: 4/12/2020 


\title{
$\cos$
}

\section{MANUSCRITOS Y HUMANISMO EN LOS LIBROS DE CABALLERÍAS: LA MATERIALIDAD EN LA FICCIÓN}

RESUMEN: El presente trabajo examina la presencia de los manuscritos, vinculados a los tópicos del manuscrito encontrado y la falsa traducción, al interior de la ficción en el género de los libros de caballerías castellanos. En particular, se estudia la caracterización y la historia de dichos objetos presentados en diversos textos de este género; es decir, se propone hacer un análisis de los rasgos que configuran la materialidad de los manuscritos que aparecen en la ficción. Así, además de atender dichos rasgos, se toma en cuenta la historia de su producción, transmisión y recepción presentados en el relato. A partir de las características de dichos manuscritos, se plantea que la ficción del género refleja muchas de la prácticas y preocupaciones centrales del Humanismo, así como la realidad material de su trabajo textual.

PAlabras-Clave: Humanismo. Manuscritos. Libros de caballerías. Materialidad. Ficción.

\section{ManusCripts AND Humanism in ChIVALRic RomanCE: MATERIALITY IN Fiction}

\begin{abstract}
This work examines manuscripts, linked to the topoi of the discovered manuscript and the pretense of translation, in the fictional worlds of romances of chivalry. Specifically, it studies the characterization and history of such manuscripts in different works of the genre. The article analyses the traits used to describe the material aspect of manuscripts in fiction. Furthermore, it takes into account the history of the manuscripts, that is, its creation, transmission, and reception, beyond their physical characteristics. All of these aspects portray a direct link with the central textual practices and concerns of Humanisms, as well as the material reality of its works.
\end{abstract}

KeYwords: Humanism. Manuscripts. Chivalric Romances. Materiality. Fiction. 